\title{
Rigidity Evaluation Model for Aircraft Structural Parts Web Based on Beam Bending Theory
}

\author{
Qinghong Gong ${ }^{1,}$, Chao Sun ${ }^{1, b}$ and Wenping Mou ${ }^{1, c}$ \\ ${ }^{1} \mathrm{NC}$ Machining Technology Research Laboratory, Chengdu Aircraft Industrial (Group) CO.LTD, \\ Chengdu, 610092 \\ anwpu_gongqh@163.com, ${ }^{\mathrm{b}}$ sun33chao@qq.com, ${ }^{\mathrm{C}}$ mouwenp@qq.com
}

\begin{abstract}
Keywords: Aircraft structural part; model of beam bending; closed web; open web; rigidity Abstract. Based on theories of beam bending, a quantitative evaluation method for web rigidity based on aircraft structural part geometrical features and process features was proposed. Combined with the characteristics of cutting force of web features in aircraft structural parts and the basic theory of simply supported beam and cantilever beam, a rigidity evaluation model for closed and open pocket bottom was set up; and a rigidity calculation model for bottom of rectangle and circle pocket was established. The machining deformation of open and closed rectangular webs was analyzed using the ABAQUS software, and the accuracy of rigidity calculation model was Validated by the simulation results. The web rigidity evaluation model can provide theoretical reference for aircraft structural part web rigidity judgment, clamping scheme and cutting parameters optimization.
\end{abstract}

\section{Introduction}

With the rapid development of the aviation industry, thin-wall monolithic components are widely used in the design of new-generation aircraft. The aviation monolithic components, with a large number of weak rigid structures like thin walls etc, are becoming more complicated and demand higher processing precision, which results in more challenges in manufacturing. The scientific evaluation of the rigidity of the parts before machining is the prerequisite for optimizing the processing plan and the cutting parameters, which plays a more prominent role in the manufacturing process of aircraft structural parts. At present, two methods are used to study the rigidity evaluation of thin-wall parts, the first is using finite element simulation software to analyze parts' deformation, then evaluate the rigidity of parts; the second method is using the optimization algorithm to establish the relationship between the clamping-parts-deformation to evaluate the rigidity of the parts.

In the research of finite element simulation, Yuan[1] simulated and analyzed the deformation of the complex thin-wall structural parts of the cars' main model, and optimized the clamping layout based on deformation control. Dong[2] simulated the influence of the clamping position, the clamping sequence and the loading method on the deformation of the frame thin-wall parts, and optimized the clamping scheme to obtain the average residual stress and reduce the part deformation. Jin[3] modeled the milling process of the arc-shaped thin-wall parts and optimized the clamping point position by considering the transient milling forces. Li[4] analyzed the effect of residual stress on the deformation during the processing of thin-wall frame parts by finite element method, preferred the varied cutting-depth milling method and inside-to-outside milling path to improve the machining efficiency and quality. Richter[5] used the measured residual stress value as the input to simulate the parts processing deformation, which required a high accuracy of the residual stress measurement. Wang[6] employed birth-death element technology and mobile loading method to simulate the material removal, and predicted the deformation of thin-wall parts.

In the study of optimization algorithms, Zheng[7] proposed a method to evaluate the rigidity of parts based on fuzzy theory, the method took account of accuracy, deformation, stability and interference et al, and established the comprehensive evaluation system of parts' rigidity, but it could not predict the rigidity of parts with clamping. Qin[8] formulated the DOFs constraining principle to describing the relationship between the machining dimensions and the DOFs, and concluded the judgment theorems for locating correctness. 
Frame-beam component is a typical aircraft structural part, having thin-wall, large dimension, complex structure. The key to evaluate the rigidity of frame-beam structural parts is to obtain the rigidity of the webs accurately.

The web is an important processing feature of the frame-beam aircraft structural parts, the rigidity of the web is an important factor to be considered in the selection of the clamping plan and the machining parameters. The web can be categorized into closed webs and open webs, as is shown in Fig 1. In this paper, structural characteristics of closed webs and open webs were studied. In addition, we established a model to evaluate the rigidity of webs based on the mechanical model of the bending and deformation of the simply supported beam and cantilever beam, which considered the effect of the cutting force vertical to the web during the milling process. Then we carried out a finite element simulation of web rigidity to verify the accuracy of the theoretical model of web rigidity evaluation.

\section{Rigid Evaluation Model for Web Based on Beam Bending Theory}

According to the theory of bending deformation, when the beam is subjected to concentrated load perpendicular to its axial direction, bending deformation occurs, and its bending rigidity can be expressed as

$$
K=E \cdot I_{z}
$$

where, $\mathrm{E}$ is the young's modulus of the material, $I_{z}$ is the inertial moment of the cross section of the beam relative to the center $\mathrm{z}$ axis which can be expressed by Eq.1.

$$
I_{z}=\int y^{2} d A
$$

If the shape of the section is rectangular and the length of the rectangle is $\mathrm{m}$ ((Fig.1, $\mathrm{z}$ direction), the width is $\mathrm{n}$ (Fig.2, $\mathrm{Y}$ direction), then the inertial moment of the cross section relative to the $\mathrm{Z}$ axis can be expressed as:

$$
I_{z}=\frac{1}{12} m n^{3}
$$

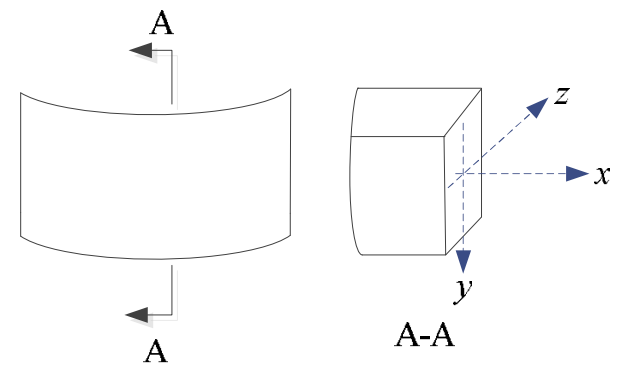

Fig. 1 A schematic diagram of beam cross-section

The deformations of beams with different confinement types are different when they are subjected to different loads in the direction perpendicular to the length of the beam, while the maximum deformation is the biggest concern in engineering applications. In general, the largest deformation takes place in the weakest place for the force of the point of action. Considering the engineering application, this paper mainly considers the simple supported beam (Fig. 2) and the cantilever beam (Fig. 3). The maximum deformation of the simply supported beam occurs at the midpoint of the length of the beam which can be represented as:

$$
\omega=\frac{F l^{3}}{48 E I}=\frac{F}{\frac{48 E I}{l^{3}}}=\frac{F}{K_{i}}
$$

The maximum deformation of the cantilever occurs at the farthest distance from the support. It can be represented as:

$$
\omega=\frac{F l^{3}}{3 E I}=\frac{F}{\frac{3 E I}{l^{3}}}=\frac{F}{K_{i}}
$$


In the Eq.4 and Eq.5, the F indicates the concentrated load on the beam; $l$ indicates the length of the cantilever beam; $\omega$ represents the maximum deformation of the beam; and $K_{i}$ indicates the flexural rigidity of the beam. Therefore, both the simply supported beam and the cantilever beam can be used to represent the weakest flexural rigidity of the beam.

It is clear that the weakest flexural rigidity of the beam is proportional to the moment of inertia of the beam $I_{z}$, and is inversely proportional to the cubic of the distance $l$ from the weakest point to the constraint point. $\mathrm{K}$ denotes the bending rigidity coefficient of the different beam model, Simple beam coefficient $k=48$, cantilever beam coefficient $\mathrm{k}=3$.
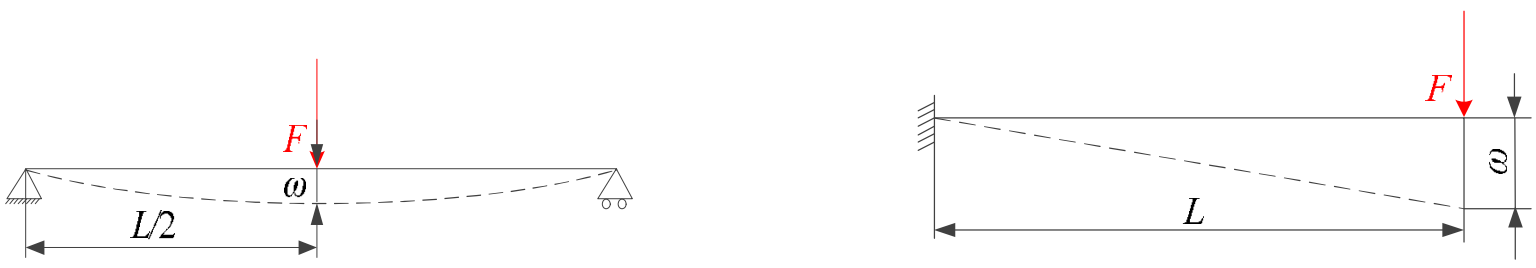

Fig. 2 A schematic diagram of simply supported beam Fig.3 A schematic diagram of cantilever beam

In the process of NC machining of the aircraft structure, the web is mainly affected by the cutting force perpendicular to the web, which can be simplified as concentrated load, while the form of the web has a variety of structural forms due to the different structure of the parts. However, as a whole, the structural characteristics of a single groove cavity web can be divided into two types: closed type (closed groove) and open type (open slot). For the two specific structures, the theoretical model is as follows

\section{Rigid evaluation of closed Web}

Fig. 4 represents a fully enclosed slot cavity structure. To simplify the problem, it can be assumed that the thickness of the bottom web plate is equal everywhere and is expressed in to simplify the problem, thickness of the bottom web plate can be assumed to be homogeneous. The weakest part of the web is the geometric center $\mathrm{O}$ in the course of its processing. In order to ensure the rigidity of the web area to meet the processing requirements, just ensure that the rigidity of the $O$ point meet the requirements, so it only need that the rigidity of the $\mathrm{O}$ point be evaluated in the engineering application.

To ensure the rigidity of the web area to meet the processing requirements, whether the rigidity of Point $\mathrm{O}$ meets the requirements or not must be ensured. Thus the evaluation of rigidity of Point $\mathrm{O}$ is required for engineering applications.

Because the web is a fully enclosed area, it can be equivalent to four complete constraints. In order to calculate the rigidity of the O-point, the web is divided into numerous simple-supported beams. The working force of the tool is perpendicular to the length of the beam. The beam is mainly subjected to the bending load. Therefore, evaluating the rigidity of the O-point is converted? converted to what? to evaluate its bending rigidity, and the bending stiffness of each section of the beam at its point $\mathrm{O}$ can be calculated first

$$
K_{\mathrm{o}}=48 \cdot \frac{E I}{l^{3}}=48 \cdot \frac{E}{l^{3}} \cdot \frac{1}{12} t^{3} \cdot \mathrm{d} s
$$

If the length of the beam can be expressed as a function of s i.e. $l(s)$, then

$$
K_{\mathrm{o}}=48 \cdot \frac{E I}{l^{3}(s)}=48 \cdot \frac{E}{l^{3}(s)} \cdot \frac{1}{12} t^{3} \cdot \mathrm{d} s
$$

The integral bending stiffness of the $\mathrm{O}$ point in the closed web region can be obtained by the integral of the web region

$$
K_{\mathrm{o}}=\frac{1}{2} \cdot \int 48 \cdot \frac{E}{l^{3}(s)} \cdot \frac{1}{12} t^{3} \cdot \mathrm{d} s
$$




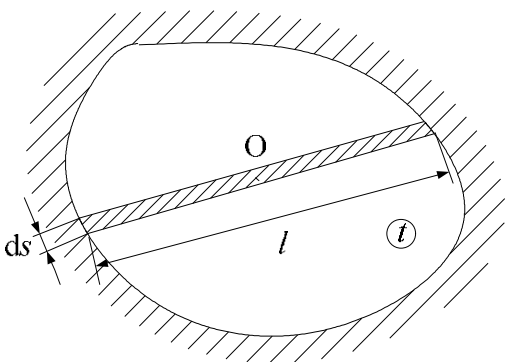

Fig. 4 The structural diagram of closed web

\section{Evaluation of open web rigidity}

The weakest rigid point $\mathrm{O}$ of the open web is somewhere in the middle of the opening (Fig. 5), If the web thickness is $t$, the length of the equivalent beam is a function of $\mathrm{s}, l(s)$. The web is treated differentially. It is equivalent to several cantilever beam bending models with $\mathrm{O}$ point as the free end. When the concentrated load acts at the $\mathrm{O}$ point, its deformation is the largest, and its flexural rigidity can be expressed as

$$
K_{\mathrm{o}}=3 \cdot \frac{E I}{l^{3}(s)}=3 \cdot \frac{E}{l^{3}(s)} \cdot \frac{1}{12} t^{3} \cdot \mathrm{d} s
$$

The flexural rigidity of the open web at the $\mathrm{O}$ point is obtained by integrating the bounded boundary (excellent arc BA)

$$
K_{\mathrm{o}}=\int_{\mathrm{BA}} 3 \cdot \frac{E}{l^{3}(s)} \cdot \frac{1}{12} t^{3} \cdot \mathrm{d} s
$$

\section{Calculation model of typical web structural features' bending stiffness}

The structural characteristics of aircraft frame beam structures are mainly rectangular or circular. Therefore, the calculation model of web rigidity is deduced with rectangular and circular structural features as concrete examples

\section{Rectangular Web}

(1) Closed type

If the length of the rectangle is a and the width is b as shown in Fig.6, taking a short beam of FGHI shown in Fig.5, the bending rigidity of the point $\mathrm{O}$ can be expressed as

$$
K_{\mathrm{o}}=48 \cdot \frac{E I}{\left[2\left(\sqrt{(a / 2)^{2}+x^{2}}\right)\right]^{3}}=48 \cdot \frac{E}{8\left({\sqrt{(a / 2)^{2}+x^{2}}}^{3}\right.} \cdot \frac{1}{12} t^{3} \cdot \mathrm{d} x=\frac{E t^{3}}{2{\sqrt{(a / 2})^{2}+x^{2}}^{3}} \cdot \mathrm{d} x
$$

Eq.11 is integrated in the illustrated ECAM area:

$$
K_{a}^{\prime}=\int_{0}^{b / 2}\left(\frac{E t^{3}}{2\left(\sqrt{(a / 2)^{2}+x^{2}}\right)^{3}}\right) \cdot \mathrm{d} x=E t^{3} \cdot \frac{2 b}{a^{2} \cdot \sqrt{a^{2}+b^{2}}}
$$

From the symmetry, the bending rigidity of the $\mathrm{O}$ point in the direction of the length (the size is the direction of a) is obtained

$$
K_{a}=2 K_{a}^{\prime}=E t^{3} \cdot \frac{4 b}{a^{2} \cdot \sqrt{a^{2}+b^{2}}}
$$

In the same way, the rigidity of the width direction of the rectangular frame can be deduced:

$$
K_{b}=2 K_{b}^{\prime}=E t^{3} \cdot \frac{4 a}{b^{2} \cdot \sqrt{a^{2}+b^{2}}}
$$

Therefore, the overall flexural rigidity of the fully enclosed rectangular frame can be expressed as

$$
K_{1}=K_{a}+K_{b}=E t^{3}\left(\frac{4 b}{a^{2} \cdot \sqrt{a^{2}+b^{2}}}+\frac{4 a}{b^{2} \cdot \sqrt{a^{2}+b^{2}}}\right)
$$




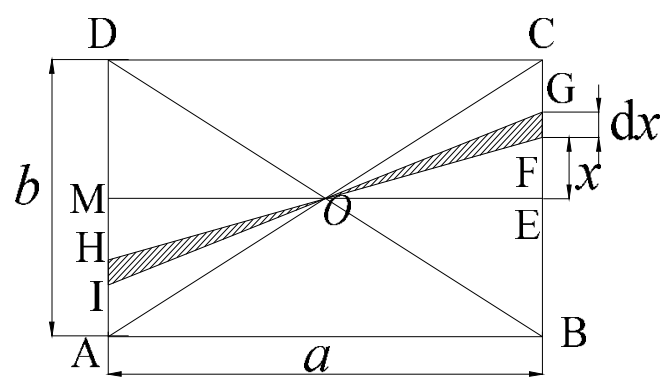

Fig. 6 closed rectangular web

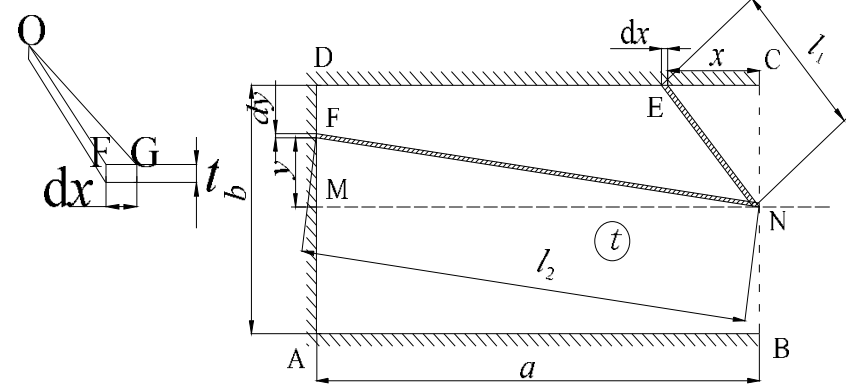

Fig. 7 open rectangular web

(2)Open type

As shown in Fig.6, a section of ABCD of the rectangular web BC opens. The thickness of the web is t. From the geometric structure, it shows that when the concentrated load acts at the midpoint $\mathrm{N}$ of the opening, the deformation of the $\mathrm{N}$ point is the largest. According to the characteristic structure of the web, the web can be differentiated into two types of cantilever beams. One is the beam EN with the length direction as the fixed end (CD direction) and the $\mathrm{N}$ point as the free end, and the other is the beam $\mathrm{FN}$ with the $\mathrm{AD}$ direction as the fixed end and the $\mathrm{N}$ point as the free end.

The flexural rigidity of the two kinds of beam $\mathrm{N}$ points can be expressed as

$$
\begin{aligned}
& K_{\mathrm{N} 1}=3 \cdot \frac{E I}{\left(\sqrt{(b / 2)^{2}+x^{2}}\right)^{3}}=3 \cdot \frac{E}{\left(\sqrt{(b / 2)^{2}+x^{2}}\right)^{3}} \cdot \frac{1}{12} t^{3} \cdot \mathrm{d} x=\frac{1}{4} \cdot \frac{E t^{3}}{\left(\sqrt{(b / 2)^{2}+x^{2}}\right)^{3}} \cdot \mathrm{d} x \\
& K_{\mathrm{N} 2}=3 \cdot \frac{E I}{\left(\sqrt{a^{2}+y^{2}}\right)^{3}}=3 \cdot \frac{E}{\left(\sqrt{\left(a^{2}+y^{2}\right.}\right)^{3}} \cdot \frac{1}{12} t^{3} \cdot \mathrm{d} y=\frac{1}{4} \cdot \frac{E t^{3}}{\left(\sqrt{a^{2}+y^{2}}\right)^{3}} \cdot \mathrm{d} y
\end{aligned}
$$

Eq.16 and Eq.17 are respectively fixed along the fixed end boundary to obtain the rigidity along the width direction and the length direction

$$
\begin{aligned}
& K_{\mathrm{N} b}=\frac{E t^{3}}{4} \cdot \int_{0}^{a} \frac{1}{\left(\sqrt{(b / 2)^{2}+x^{2}}\right)^{3}} \cdot \mathrm{d} x=\frac{E t^{3}}{4} \cdot \frac{8 a}{b^{2} \cdot \sqrt{4 a^{2}+b^{2}}}=E t^{3} \cdot \frac{2 a}{b^{2} \cdot \sqrt{4 a^{2}+b^{2}}} \\
& K_{\mathrm{N} a}=\frac{E t^{3}}{4} \cdot \int_{0}^{b / 2} \frac{1}{\left(\sqrt{a^{2}+y^{2}}\right)^{3}} \cdot \mathrm{d} y=\frac{E t^{3}}{4} \cdot \frac{b}{a^{2} \cdot \sqrt{4 a^{2}+b^{2}}}
\end{aligned}
$$

It is known by symmetry that the bending rigidity of $\mathrm{N}$ point of open rectangular web can be expressed as

$$
K_{\mathrm{N}}=2 K_{\mathrm{N} b}+2 K_{\mathrm{N} a}=E t^{3} \cdot\left(\frac{4 a}{b^{2} \cdot \sqrt{4 a^{2}+b^{2}}}+\frac{b}{2 a^{2} \cdot \sqrt{4 a^{2}+b^{2}}}\right)
$$

\section{Round web}

\section{(1)Closed type}

If $\mathrm{R}$ is the radius of the circle, consider a simple beam of ABCD as shown in Fig.9. The flexural rigidity of the point $\mathrm{O}$ can be expressed as

$$
K_{\mathrm{o}}=48 \cdot \frac{E I}{(2 R)^{3}}=48 \cdot \frac{E}{8 R^{3}} \cdot \frac{1}{12} t^{3} \cdot \mathrm{d} s=\frac{E t^{3}}{2 R^{3}} \cdot \mathrm{d} s
$$
21.

The bending strength of the closed circular web is obtained by the integral of the right end of Eq.

$$
K_{2}=\int_{\mathrm{BD}} \frac{E t^{3}}{2 R^{3}} \mathrm{~d} s=\int_{0}^{\pi} \frac{E t^{3}}{2 R^{3}} R \mathrm{~d} \theta=\frac{\pi E t^{3}}{2 R^{2}}
$$



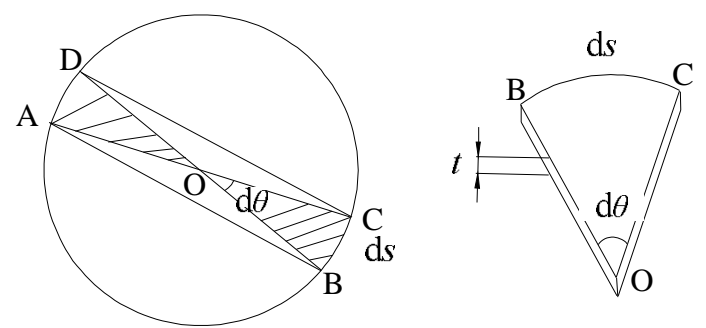

Fig. 8 The structural diagram of closed circular web

(2) Open type

As shown in Fig. 9, assuming that the open portion of the circular web is the inferior arc MN, it is known from the symmetry that the weakest point of the web is the midpoint $A$ of the inferior arc $M N$, the web thickness is $t$, the radius of the arc is R, taking the cantilever of the shadow part of the ABCD, from the geometric relationship, the length of the section beam is:

$$
l=2 R \cdot \sin \frac{\theta}{2}
$$

When the concentrated load acts on the A point, the deformation is the maximum, and the flexural rigidity of beam $\mathrm{ABCD}$ at $\mathrm{A}$ can be expressed as

$$
K_{\mathrm{A}}=3 \cdot \frac{E I}{l^{3}(s)}=3 \cdot \frac{E}{\left(2 R \cdot \sin \frac{\theta}{2}\right)^{3}} \cdot \frac{1}{12} t^{3} \cdot \mathrm{d} s
$$

Eq.24 is integrated along the superior arc NM to obtain the bending rigidity of the open web at point A by the concentrated load

$$
K_{3}=\frac{E t^{3}}{12 R^{2}} \int_{\alpha / 2}^{2 \pi-\alpha / 2} \frac{\mathrm{d}\left(\frac{\theta}{2}\right)}{\left(\sin \frac{\theta}{2}\right)^{3}}=\left[-\frac{1}{2} \cdot \frac{\cos \frac{\theta}{2}}{\sin ^{2} \frac{\theta}{2}}+\frac{1}{2} \cdot \ln \left|\tan \frac{\theta}{4}\right|\right]_{\frac{\alpha}{2}}^{2 \pi-\frac{\alpha}{2}}
$$

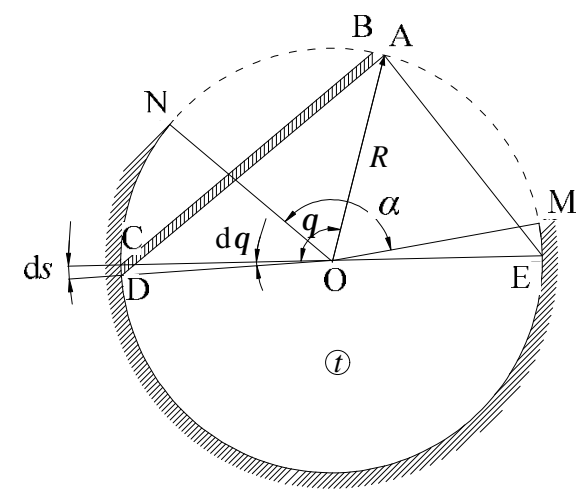

Fig. 9 The structural diagram of open circular web
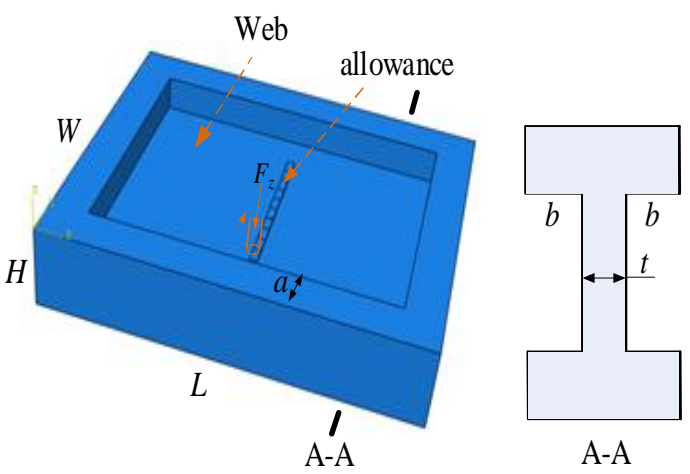

Fig.10 the closed rectangle web

\section{FEM-simulation}

To validate verified the accuracy of the rigidity evaluation model for aircraft structural parts web, a case study of rectangle web using ABAQUS to structure the FEM of different thickness is set up. Using ABAQUS to construct Finite element model of varying thickness is setup. The death cell of ABAQUS is used to define the removal of allowance, meanwhile apply the load of moving cutting force load of moving cutting force is applied to simulate the deformation of the web.

Fig.11 and Fig.12 show the size of closed and open rectangle web in the simulation, the size of web defined as $\mathrm{L}=250 \mathrm{~mm}, \mathrm{~W}=200 \mathrm{~mm}, \mathrm{H}=2 \mathrm{~b}+\mathrm{t}, \mathrm{a}=\mathrm{b}=20 \mathrm{~mm}, \mathrm{t}=12 \mathrm{~mm} 、 10 \mathrm{~mm} 、 8 \mathrm{~mm} 、 6 \mathrm{~mm} 、 4 \mathrm{~mm}$, allowance is $a p=2 \mathrm{~mm}$, ae $=5 \mathrm{~mm}$ 。 


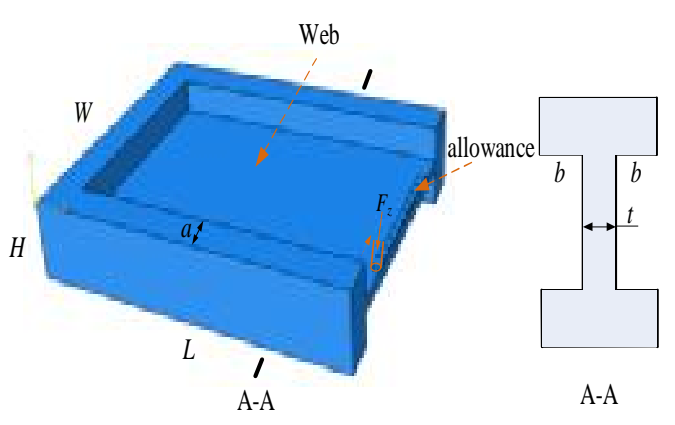

Fig.11 the open rectangle web model

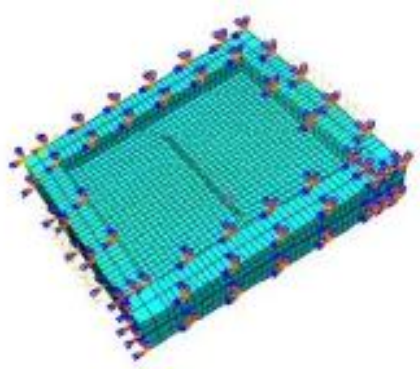

Fig.12 the load and boundary of the simulation

The boundary conditions and load applied in the simulation are shown in Fig.12, the fixed restriction is loaded on the stiffener connected with the web, meshing the chipping allowance and defined the mesh property by the death cell. Simulating the process of remove the cutting cushion while apply cutting force load on the death cell, the cutting load moved with the removal of chipping allowance.The mesh is C3D20R, the workpiece is aluminium alloy 7050-T7451, the stress-strain relationship is defined by the model of Johnson-cook..

The diagram of machining deformation on closed wed is shown in Fig.13, the distortion of the wed increased firstly and then decreases with the moving of tool. The biggest deformation occurred when the tool cutting the middle allowance of the wed, which is $0.551 \mathrm{~mm}$ in the $\mathrm{Z}$ direction.
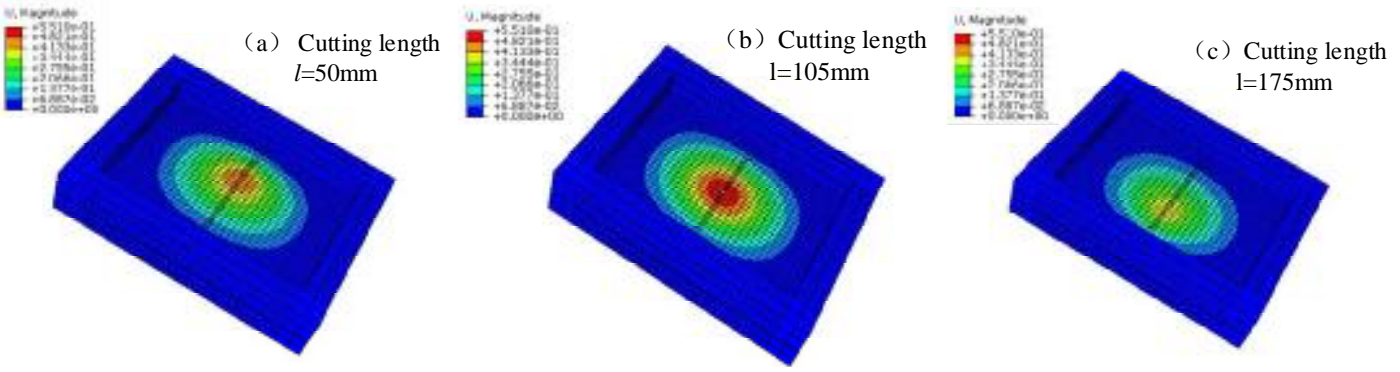

Fig.13 diagram of machining deformation on closed wed

The diagram of machining deformation on open wed is shown in Fig. 14, the biggest deformation is $1.390 \mathrm{~mm}$ in the $\mathrm{Z}$ direction.
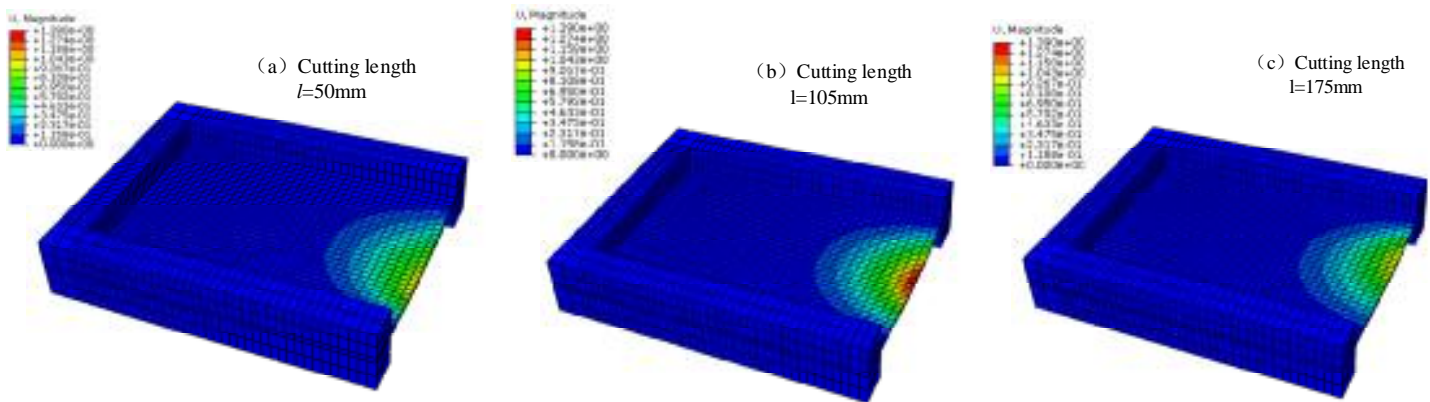

Fig.14 diagram of machining deformation on open wed

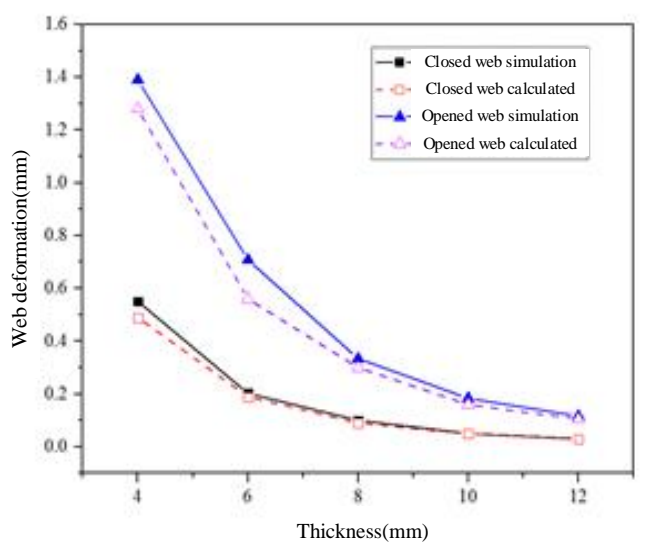

Fig.15 curve of comparison between simulation and calculation 
Fig. 15 shows the comparison between simulation and the theoretical model of rigidity evaluation mentioned above.Comparing the result of simulation and rigid evaluation, the weakest position is consistent in simulation and evaluation, the deviation is less than $10 \%$. So that the Rigid evaluation model can evaluate the machining distortion accurately, direct the establishment and implementation of processing plan.

\section{Conclusions}

The frame beam parts of plane has complicated structure and weak rigidity, which take difficulties for fixture design and the development of technology programs. Determination of rigid parts strongly depends on artificial experience, the engineering feasibility is unacceptable. Therefore a rigid evaluation model for aircraft Structural parts web based on beam bending theory is suggested in this paper. The bending rigidity of closed and open web is achieved, and rectangle and circular web were considered to calculate the rigidity, which provides a theoretical reference for determining the part integral rigidity, optimizing clamping scheme and selecting cutting parameters. Following conclusions can be drawn from this paper:

(1) Utilizing the feature of web, based on the model of beam bending theory, and combining the mechanical model with engineering practice, the theory model of rigid evaluation is established, and then a method for accurate evaluation of web rigidity was achieved.

(2) A general computation method of closed and open web is given.

(3) Taking rectangle and circular web for example, a calculation equation of rigidity on web is given.

\section{Acknowledgements}

This work was financially supported by the National Science and Technology Major Project. (No. 2015ZX04001-002).

\section{References}

[1] YUAN J S. Finite element simulation of deformation and fixture layout optimization in milling of complex thin-walled parts[D]. Shanghai Jiao Tong university, 2011 (in Chinese).

[2] DONG H Y, KE Y L. Finite element simulation for optimal clamping scheme of thin-walled workpiece in milling process[J]. Journal of Zhejiang University (Engineering Science), 2004, 38(1): 18-21 (in Chinese)

[3] JIN Q, LIU S G. Optimization of fixture scheme for milling thin-walled arc workpiece[J]. Tool Engineering, 2007, 12: 54-57 (in Chinese).

[4] LI K, LI P Z, YANG J G, et al. Research on the processing method and controlling of distortion for the thin-walled weak rigidity parts[J]. Modular Machine Tool \& Automatic Manufacturing Technique, 2013 (10): 101-104 (in Chinese).

[5] Richter-Trummer V, Koch D, Witte A, et al. Methodology for prediction of distortion of workpieces manufactured by high speed machining based on an accurate through-the-thickness residual stress determination[J]. The International Journal of Advanced Manufacturing Technology, 2013, 68(9-12): 2271-2281.

[6] WANG Z H, YUAN J T, LIU T T, et al. Machining deformation in birth-death element for thin-walled workpiece[J]. Journal of Harbin University of Science and Technology, 2013, 17(6): 81-85 (in Chinese).

[7] ZHENG Z Y, XIE M, WANG S C. Fuzzy synthetic evaluation for fixturing solution[J]. Aeronautical manufacturing technology, 2001,2:31-33 (in Chinese).

[8] QIN G H, ZHANG W H, LI Y L. A new algorithm of fixture locating scheme design[J]. Journal of test and measurement technology, 2008, 22(3): 236-240 (in Chinese). 